\title{
A sharp inequality for multilinear singular integral operators with non-smooth kernels
}

Guangze $\mathrm{Gu}^{*}$ and Mingjie Cai

"Correspondence: to_ggz@163.com College of Mathematics and Econometrics, Hunan University, Changsha, 410082, P.R. China

\begin{abstract}
In this paper, we establish a sharp inequality for some multilinear singular integral operators with non-smooth kernels. As an application, we obtain the weighted $L^{p}$-norm inequality and $L \log L$-type inequality for the multilinear operators.

MSC: 42B20; 42B25

Keywords: multi-linear operator; singular integral operator with non-smooth kernel; sharp inequality; $\mathrm{BMO} ; A_{p}$-weight
\end{abstract}

\section{Definitions and results}

As the development of singular integral operators and their commutators, multilinear singular integral operators have been well studied (see [1-6]). In this paper, we study some multilinear operator associated to the singular integral operators with non-smooth kernels as follows.

Definition 1 A family of operators $D_{t}, t>0$, is said to be 'approximations to the identity' if, for every $t>0, D_{t}$ can be represented by the kernel $a_{t}(x, y)$ in the following sense:

$$
D_{t}(f)(x)=\int_{R^{n}} a_{t}(x, y) f(y) d y
$$

for every $f \in L^{p}\left(R^{n}\right)$ with $p \geq 1$, and $a_{t}(x, y)$ satisfies

$$
\left|a_{t}(x, y)\right| \leq h_{t}(x, y)=C t^{-n / 2} s\left(|x-y|^{2} / t\right),
$$

where $s$ is a positive, bounded and decreasing function satisfying

$$
\lim _{r \rightarrow \infty} r^{n+\epsilon} s\left(r^{2}\right)=0
$$

for some $\epsilon>0$.

Definition 2 A linear operator $T$ is called a singular integral operator with non-smooth kernel if $T$ is bounded on $L^{2}\left(R^{n}\right)$ and associated with a kernel $K(x, y)$ such that

$$
T(f)(x)=\int_{R^{n}} K(x, y) f(y) d y
$$

(C) 2013 Gu and Cai; licensee Springer. This is an Open Access article distributed under the terms of the Creative Commons Attribution License (http://creativecommons.org/licenses/by/2.0), which permits unrestricted use, distribution, and reproduction in any medium, provided the original work is properly cited. 
for every continuous function $f$ with compact support, and for almost all $x$ not in the support of $f$.

(1) There exists an 'approximation to the identity' $\left\{B_{t}, t>0\right\}$ such that $T B_{t}$ has an associated kernel $k_{t}(x, y)$ and there exist $c_{1}, c_{2}>0$ so that

$$
\int_{|x-y|>c_{1} t^{1 / 2}}\left|K(x, y)-k_{t}(x, y)\right| d x \leq c_{2} \quad \text { for all } y \in R^{n} .
$$

(2) There exists an 'approximation to the identity' $\left\{A_{t}, t>0\right\}$ such that $A_{t} T$ has an associated kernel $K_{t}(x, y)$ which satisfies

$$
\left|K_{t}(x, y)\right| \leq c_{4} t^{-n / 2} \quad \text { if }|x-y| \leq c_{3} t^{1 / 2}
$$

and

$$
\left|K(x, y)-K_{t}(x, y)\right| \leq c_{4} t^{\delta / 2}|x-y|^{-n-\delta} \quad \text { if }|x-y| \geq c_{3} t^{1 / 2}
$$

for some $c_{3}, c_{4}>0, \delta>0$.

Let $m_{j}$ be positive integers $(j=1, \ldots, l), m_{1}+\cdots+m_{l}=m$, and let $b_{j}$ be functions on $R^{n}$ $(j=1, \ldots, l)$. Set, for $1 \leq j \leq m$,

$$
R_{m_{j}+1}\left(b_{j} ; x, y\right)=b_{j}(x)-\sum_{|\alpha| \leq m_{j}} \frac{1}{\alpha !} D^{\alpha} b_{j}(y)(x-y)^{\alpha} .
$$

The multilinear operator associated to $T$ is defined by

$$
T^{b}(f)(x)=\int_{R^{n}} \frac{\prod_{j=1}^{l} R_{m_{j}+1}\left(b_{j} ; x, y\right)}{|x-y|^{m}} K(x, y) f(y) d y .
$$

Note that when $m=0, T^{b}$ is just the multilinear commutator of $T$ and $b_{j}$ (see [7]). However, when $m>0, T_{b}$ is a non-trivial generalization of the commutator. It is well known that multilinear operators are of great interest in harmonic analysis and have been widely studied by many authors (see [1-4]). Hu and Yang (see [8]) proved a variant sharp estimate for the multilinear singular integral operators. In [7], Pérez and TrujilloGonzalez proved a sharp estimate for the multilinear commutator when $b_{j} \in O s c_{\exp L^{r_{j}}}\left(R^{n}\right)$ and noted that $O s c_{\exp L^{r_{j}}} \subset B M O$. The main purpose of this paper is to prove a sharp function inequality for the multilinear singular integral operator with non-smooth kernel when $D^{\alpha} b_{j} \in B M O\left(R^{n}\right)$ for all $\alpha$ with $|\alpha|=m_{j}$. As an application, we obtain an $L^{p}(p>1)$ norm inequality and an $L \log L$-type inequality for the multilinear operators. In [9-12], the boundedness of a singular integral operator with non-smooth kernel is obtained. In [13], the boundedness of the commutator associated to the singular integral operator with non-smooth kernel is obtained. Our works are motivated by these papers.

First, let us introduce some notations. Throughout this paper, $Q$ denotes a cube of $R^{n}$ with sides parallel to the axes. For any locally integrable function $f$, the sharp function of $f$ is defined by

$$
f^{\#}(x)=\sup _{Q \ni x} \frac{1}{|Q|} \int_{Q}\left|f(y)-f_{Q}\right| d y,
$$


where, and in what follows, $f_{Q}=|Q|^{-1} \int_{Q} f(x) d x$. It is well known that (see $\left.[14,15]\right)$

$$
f^{\#}(x) \approx \sup _{Q \ni x} \inf _{\mathbf{C} \in \mathbf{C}} \frac{1}{|Q|} \int_{Q}|f(y)-c| d y .
$$

We say that $f$ belongs to $B M O\left(R^{n}\right)$ if $f^{\#}$ belongs to $L^{\infty}\left(R^{n}\right)$ and $\|f\|_{B M O}=\left\|f^{\#}\right\|_{L^{\infty}}$. Let $M$ be a Hardy-Littlewood maximal operator defined by

$$
M(f)(x)=\sup _{Q \ni x} \frac{1}{|Q|} \int_{Q}|f(y)| d y .
$$

For $k \in N$, we denote by $M^{k}$ the operator $M$ iterated $k$ times, i.e., $M^{1}(f)(x)=M(f)(x)$ and

$$
M^{k}(f)(x)=M\left(M^{k-1}(f)\right)(x) \quad \text { when } k \geq 2 .
$$

The sharp maximal function $M_{A}(f)$ associated with the 'approximations to the identity' $\left\{A_{t}, t>0\right\}$ is defined by

$$
M_{A}^{\#}(f)(x)=\sup _{Q \ni x} \frac{1}{|Q|} \int_{Q}\left|f(y)-A_{t_{Q}}(f)(y)\right| d y,
$$

where $t_{Q}=l(Q)^{2}$ and $l(Q)$ denotes the side length of $Q$. For $0<r<\infty$, we denote $M_{A}^{\#}(f)_{r}$ by

$$
M_{A}^{\#}(f)_{r}=\left[M_{A}^{\#}\left(|f|^{r}\right)\right]^{1 / r} .
$$

Let $\Phi$ be a Young function and $\tilde{\Phi}$ be the complementary associated to $\Phi$. For a func$\operatorname{tion} f$, we denote the $\Phi$-average by

$$
\|f\|_{\Phi, Q}=\inf \left\{\lambda>0: \frac{1}{|Q|} \int_{Q} \Phi\left(\frac{|f(y)|}{\lambda}\right) d y \leq 1\right\}
$$

and the maximal function associated to $\Phi$ by

$$
M_{\Phi}(f)(x)=\sup _{Q \ni x}\|f\|_{\Phi, Q} .
$$

The Young functions used in this paper are $\Phi(t)=t(1+\log t)^{r}$ and $\tilde{\Phi}(t)=\exp \left(t^{1 / r}\right)$, the corresponding average and maximal functions are denoted by $\|\cdot\|_{L(\log L)^{r}, Q}, M_{L(\log L)^{r}}$ and $\|\cdot\|_{\exp L^{1 / r}, Q}, M_{\exp L^{1 / r}}$. Following $[11,12,16]$, we know the generalized Hölder inequality

$$
\frac{1}{|Q|} \int_{Q}|f(y) g(y)| d y \leq\|f\|_{\Phi, Q}\|g\|_{\tilde{\Phi}, Q}
$$

and the following inequality, for $r, r_{j} \geq 1, j=1, \ldots, l$ with $1 / r=1 / r_{1}+\cdots+1 / r_{l}$, and any $x \in R^{n}, b \in B M O\left(R^{n}\right)$,

$$
\begin{aligned}
& \|f\|_{L(\log L)^{1 / r, Q}} \leq M_{L(\log L)^{1 / r}}(f) \leq C M_{L(\log L)^{l}}(f) \leq C M^{l+1}(f), \\
& \left\|b-b_{Q}\right\|_{\exp L^{r}, Q} \leq C\|b\|_{B M O}, \\
& \left|b_{2^{k+1} Q}-b_{2 Q}\right| \leq C k\|b\|_{B M O} .
\end{aligned}
$$

We denote the Muckenhoupt weights by $A_{p}$ for $1 \leq p<\infty$ (see [14]). 
We shall prove the following theorems.

Theorem 1 If $T$ is a singular integral operator with non-smooth kernel as given in Definition 2, let $D^{\alpha} b_{j} \in B M O\left(R^{n}\right)$ for all $\alpha$ with $|\alpha|=m_{j}$ and $j=1, \ldots$, l. Then there exists a constant $C>0$ such that for any $f \in C_{0}^{\infty}\left(R^{n}\right), 0<r<1$ and $\tilde{x} \in R^{n}$,

$$
M_{A}^{\#}\left(T^{b}(f)\right)_{r}(\tilde{x}) \leq C \prod_{j=1}^{l}\left(\sum_{\left|\alpha_{j}\right|=m_{j}}\left\|D^{\alpha_{j}} b_{j}\right\|_{B M O}\right) M^{l+1}(f)(\tilde{x}) .
$$

Theorem 2 If $T$ is a singular integral operator with non-smooth kernel as given in Definition 2 , let $D^{\alpha} b_{j} \in B M O\left(R^{n}\right)$ for all $\alpha$ with $|\alpha|=m_{j}$ and $j=1, \ldots, l$. Then $T^{b}$ is bounded on $L^{p}(w)$ for any $1<p<\infty$ and $w \in A_{p}$, that is,

$$
\left\|T^{b}(f)\right\|_{L^{p}(w)} \leq C \prod_{j=1}^{l}\left(\sum_{\left|\alpha_{j}\right|=m_{j}}\left\|D^{\alpha_{j}} b_{j}\right\|_{B M O}\right)\|f\|_{L^{p}(w)} .
$$

Theorem 3 If $T$ is a singular integral operator with non-smooth kernel as given in Definition 2 , let $w \in A_{1}, D^{\alpha} b_{j} \in B M O\left(R^{n}\right)$ for all $\alpha$ with $|\alpha|=m_{j}$ and $j=1, \ldots, l$. Then there exists a constant $C>0$ such that for all $\lambda>0$,

$$
w\left(\left\{x \in R^{n}:\left|T^{b}(f)(x)\right|>\lambda\right\}\right) \leq C \int_{R^{n}} \frac{|f(x)|}{\lambda}\left[1+\log ^{+}\left(\frac{|f(x)|}{\lambda}\right)\right]^{l} w(x) d x .
$$

\section{Proof of the theorem}

To prove the theorems, we need the following lemma.

Lemma 1 (see [1]) Let $b$ be a function on $R^{n}$ and $D^{\alpha} b \in L^{q}\left(R^{n}\right)$ for all $\alpha$ with $|\alpha|=m$ and some $q>n$. Then

$$
\left|R_{m}(b ; x, y)\right| \leq C|x-y|^{m} \sum_{|\alpha|=m}\left(\frac{1}{|\tilde{Q}(x, y)|} \int_{\tilde{Q}(x, y)}\left|D^{\alpha} b(z)\right|^{q} d z\right)^{1 / q},
$$

where $\tilde{Q}$ is the cube centered at $x$ and having side length $5 \sqrt{n}|x-y|$.

Lemma 2 ([14, p.485]) Let $0<p<q<\infty$ and for any function $f \geq 0$, we define that for $1 / r=1 / p-1 / q$,

$$
\|f\|_{W L^{q}}=\sup _{\lambda>0} \lambda\left|\left\{x \in R^{n}: f(x)>\lambda\right\}\right|^{1 / q}, \quad N_{p, q}(f)=\sup _{E}\left\|f \chi_{E}\right\|_{L^{p}} /\left\|\chi_{E}\right\|_{L^{r}},
$$

where the sup is taken for all measurable sets $E$ with $0<|E|<\infty$. Then

$$
\|f\|_{W L^{q}} \leq N_{p, q}(f) \leq(q /(q-p))^{1 / p}\|f\|_{W L^{q}} .
$$

Lemma 3 (see [17]) Let $r_{j} \geq 1$ for $j=1, \ldots, l$, we denote that $1 / r=1 / r_{1}+\cdots+1 / r_{l}$. Then

$$
\frac{1}{|Q|} \int_{Q}\left|f_{1}(x) \cdots f_{l}(x) g(x)\right| d x \leq\|f\|_{\exp L^{r_{1}, Q}} \cdots\|f\|_{\exp L^{r_{l}, Q},}\|g\|_{L(\log L)^{1 / r}, Q} .
$$


Lemma $4([9,10])$ Let $T$ be a singular integral operator with non-smooth kernel as given in Definition 2. Then $T$ is bounded on $L^{p}\left(R^{n}\right)$ for every $1<p<\infty$ and bounded from $L^{1}\left(R^{n}\right)$ to $W L^{1}\left(R^{n}\right)$.

Lemma $5($ see $[9,12])$ For any $\gamma>0$, there exists a constant $C>0$ independent of $\gamma$ such that

$$
\left|\left\{x \in R^{n}: M(f)(x)>D \lambda, M_{A}^{\#}(f)(x) \leq \gamma \lambda\right\}\right| \leq C \gamma\left|\left\{x \in R^{n}: M(f)(x)>\lambda\right\}\right|
$$

for $\lambda>0$, where $D$ is a fixed constant which only depends on $n$. Thus

$$
\|M(f)\|_{L^{p}} \leq C\left\|M_{A}^{\#}(f)\right\|_{L^{p}}
$$

for every $f \in L^{p}\left(R^{n}\right), 1<p<\infty$.

Lemma 6 Let $\left\{A_{t}, t>0\right\}$ be an 'approximation to the identity' and $b \in B M O\left(R^{n}\right)$. Then, for every $f \in L^{p}\left(R^{n}\right), p>1$ and $\tilde{x} \in R^{n}$,

$$
\sup _{Q \ni \tilde{x}} \frac{1}{|Q|} \int_{Q}\left|A_{t_{Q}}\left(\left(b-b_{Q}\right) f\right)(x)\right| d x \leq C\|b\|_{B M O} M^{2}(f)(\tilde{x}),
$$

where $t_{Q}=l(Q)^{2}$ and $l(Q)$ denotes the side length of $Q$.

Proof We write, for any cube $\mathrm{Q}$ with $\tilde{x} \in Q$,

$$
\begin{aligned}
\frac{1}{|Q|} \int_{Q}\left|A_{t_{Q}}\left(\left(b-b_{Q}\right) f\right)(x)\right| d x \leq & \frac{1}{|Q|} \int_{Q} \int_{R^{n}} h_{t_{Q}}(x, y)\left|\left(b(y)-b_{Q}\right) f(y)\right| d y d x \\
\leq & \frac{1}{|Q|} \int_{Q} \int_{2 Q} h_{t_{Q}}(x, y)\left|\left(b(y)-b_{Q}\right) f(y)\right| d y d x \\
& +\sum_{k=1}^{\infty} \frac{1}{|Q|} \int_{Q} \int_{2^{k+1} Q \backslash 2^{k} Q} h_{t_{Q}}(x, y)\left|\left(b(y)-b_{Q}\right) f(y)\right| d y d x \\
= & I_{1}+I_{2} .
\end{aligned}
$$

We have, by the generalized Hölder inequality,

$$
\begin{aligned}
I_{1} & \leq C \frac{1}{|Q \|| 2 Q \mid} \int_{Q} \int_{2 Q}\left|\left(b(y)-b_{Q}\right) f(y)\right| d y d x \\
& \leq C\left\|b-b_{Q}\right\|_{\exp L, 2 Q}\|f\|_{L(\log L), 2 Q} \\
& \leq C\|b\|_{B M O} M^{2}(f)(\tilde{x}) .
\end{aligned}
$$

For $I_{2}$, notice for $x \in Q$ and $y \in 2^{k+1} Q \backslash 2^{k} Q$, then $|x-y| \geq 2^{k-1} t_{Q}$ and $h_{t_{Q}}(x, y) \leq C \frac{s\left(2^{2(k-1)}\right)}{|Q|}$, then

$$
\begin{aligned}
I_{2} & \leq C \sum_{k=1}^{\infty} s\left(2^{2(k-1)}\right) \frac{1}{|Q|^{2}} \int_{Q} \int_{2^{k+1} Q}\left|\left(b(y)-b_{Q}\right) f(y)\right| d y d x \\
& \leq C \sum_{k=1}^{\infty} 2^{k n} s\left(2^{2(k-1)}\right) \frac{1}{\left|2^{k+1} Q\right|} \int_{2^{k+1} Q}\left|\left(b(y)-b_{Q}\right) f(y)\right| d y
\end{aligned}
$$




$$
\begin{aligned}
& \leq C \sum_{k=1}^{\infty} 2^{k n} s\left(2^{2(k-1)}\right)\left\|b-b_{Q}\right\|_{\exp L, 2^{k+1} Q}\|f\|_{L(\log L), 2^{k+1} Q} \\
& \leq C \sum_{k=1}^{\infty} 2^{(k-1) n} s\left(2^{2(k-1)}\right)\|b\|_{B M O} M^{2}(f)(\tilde{x}) \\
& \leq C\|b\|_{B M O} M^{2}(f)(\tilde{x}),
\end{aligned}
$$

where the last inequality follows from

$$
\sum_{k=1}^{\infty} 2^{(k-1) n} s\left(2^{2(k-1)}\right) \leq C \sum_{k=1}^{\infty} 2^{-(k-1) \varepsilon}<\infty
$$

for some $\epsilon>0$. This completes the proof.

Proof of Theorem 1 It suffices to prove for $f \in C_{0}^{\infty}\left(R^{n}\right)$ and some constant $C_{0}$ that the following inequality holds:

$$
\left(\frac{1}{|Q|} \int_{Q} \|\left. T^{b}(f)(x)\right|^{r}-\left|A_{t_{Q}} T^{b}(f)(x)\right|^{r} \mid d x\right)^{1 / r} \leq C \prod_{j=1}^{l}\left(\sum_{\left|\alpha_{j}\right|=m_{j}}\left\|D^{\alpha_{j}} b_{j}\right\|_{B M O}\right) M^{l+1}(f)(x) .
$$

Without loss of generality, we may assume $l=2$. Fix a cube $Q=Q\left(x_{0}, d\right)$ and $\tilde{x} \in Q$. Let $\tilde{Q}=$ $5 \sqrt{n} Q$ and $\tilde{b}_{j}(x)=b_{j}(x)-\sum_{|\alpha|=m_{j}} \frac{1}{\alpha !}\left(D^{\alpha} b_{j}\right)_{\tilde{Q}^{x^{\alpha}}}$, then $R_{m_{j}}\left(b_{j} ; x, y\right)=R_{m_{j}}\left(\tilde{b}_{j} ; x, y\right)$ and $D^{\alpha} \tilde{b}_{j}=$ $D^{\alpha} b_{j}-\left(D^{\alpha} b_{j}\right)_{\tilde{Q}}$ for $|\alpha|=m_{j}$. We write, for $f=f \chi_{\tilde{Q}}+f \chi_{R^{n} \backslash \tilde{Q}}=f_{1}+f_{2}$,

$$
\begin{aligned}
T^{b}(f)(x)= & \int_{R^{n}} \frac{\prod_{j=1}^{2} R_{m_{j}+1}\left(\tilde{b}_{j} ; x, y\right)}{|x-y|^{m}} K(x, y) f(y) d y=\int_{R^{n}} \frac{\prod_{j=1}^{2} R_{m_{j}}\left(\tilde{b}_{j} ; x, y\right)}{|x-y|^{m}} K(x, y) f_{1}(y) d y \\
& -\sum_{\left|\alpha_{1}\right|=m_{1}} \frac{1}{\alpha_{1} !} \int_{R^{n}} \frac{R_{m_{2}}\left(\tilde{b}_{2} ; x, y\right)(x-y)^{\alpha_{1}} D^{\alpha_{1}} \tilde{b}_{1}(y)}{|x-y|^{m}} K(x, y) f_{1}(y) d y \\
& -\sum_{\left|\alpha_{2}\right|=m_{2}} \frac{1}{\alpha_{2} !} \int_{R^{n}} \frac{R_{m_{1}}\left(\tilde{b}_{1} ; x, y\right)(x-y)^{\alpha_{2}} D^{\alpha_{2}} \tilde{b}_{2}(y)}{|x-y|^{m}} K(x, y) f_{1}(y) d y \\
& +\sum_{\left|\alpha_{1}\right|=m_{1},\left|\alpha_{2}\right|=m_{2}} \frac{1}{\alpha_{1} ! \alpha_{2} !} \int_{R^{n}} \frac{(x-y)^{\alpha_{1}+\alpha_{2}} D^{\alpha_{1}} \tilde{b}_{1}(y) D^{\alpha_{2}} \tilde{b}_{2}(y)}{|x-y|^{m}} K(x, y) f_{1}(y) d y \\
& +\int_{R^{n}} \frac{\prod_{j=1}^{2} R_{m_{j}+1}\left(\tilde{b}_{j} ; x, y\right)}{|x-y|^{m}} K(x, y) f_{2}(y) d y \\
= & T\left(\frac{\prod_{j=1}^{2} R_{m_{j}}\left(\tilde{b}_{j} ; x, \cdot\right)}{|x-\cdot|^{m}} f_{1}\right)-T\left(\sum_{\left|\alpha_{1}\right|=m_{1}} \frac{1}{\alpha_{1} !} \frac{R_{m_{2}}\left(\tilde{b}_{2} ; x, \cdot\right)(x-\cdot)^{\alpha_{1}} D^{\alpha_{1}} \tilde{b}_{1}}{|x-\cdot|^{m}} f_{1}\right) \\
& -T\left(\sum_{\left|\alpha_{2}\right|=m_{2}} \frac{1}{\alpha_{2} !} \frac{R_{m_{1}}\left(\tilde{b}_{1} ; x, \cdot\right)(x-\cdot)^{\alpha_{2}} D^{\alpha_{2}} \tilde{b}_{2}}{|x-\cdot|^{m}} f_{1}\right) \\
& +T\left(\sum_{\left|\alpha_{1}\right|=m_{1},\left|\alpha_{2}\right|=m_{2}} \frac{1}{\alpha_{1} ! \alpha_{2} !} \frac{(x-\cdot)^{\alpha_{1}+\alpha_{2}} D^{\alpha_{1}} \tilde{b}_{1} D^{\alpha_{2}} \tilde{b}_{2}}{|x-\cdot|^{m}} f_{1}\right) \\
& +T\left(\frac{\prod_{j=1}^{2} R_{m_{j}+1}\left(\tilde{b}_{j} ; x, \cdot\right)}{\mid x-\cdot f^{m}} f_{2}\right)
\end{aligned}
$$


Gu and Cai Journal of Inequalities and Applications 2013, 2013:439

Page 7 of 14

and

$$
\begin{aligned}
A_{t_{Q}} T^{b}(f)(x)= & \int_{R^{n}} \frac{\prod_{j=1}^{2} R_{m_{j}}\left(\tilde{b}_{j} ; x, y\right)}{|x-y|^{m}} K_{t}(x, y) f_{1}(y) d y \\
& -\sum_{\left|\alpha_{1}\right|=m_{1}} \frac{1}{\alpha_{1} !} \int_{R^{n}} \frac{R_{m_{2}}\left(\tilde{b}_{2} ; x, y\right)(x-y)^{\alpha_{1}} D^{\alpha_{1}} \tilde{b}_{1}(y)}{|x-y|^{m}} K_{t}(x, y) f_{1}(y) d y \\
& -\sum_{\left|\alpha_{2}\right|=m_{2}} \frac{1}{\alpha_{2} !} \int_{R^{n}} \frac{R_{m_{1}}\left(\tilde{b}_{1} ; x, y\right)(x-y)^{\alpha_{2}} D^{\alpha_{2}} \tilde{b}_{2}(y)}{|x-y|^{m}} K_{t}(x, y) f_{1}(y) d y \\
& +\sum_{\left|\alpha_{1}\right|=m_{1},\left|\alpha_{2}\right|=m_{2}} \frac{1}{\alpha_{1} ! \alpha_{2} !} \int_{R^{n}} \frac{(x-y)^{\alpha_{1}+\alpha_{2}} D^{\alpha_{1}} \tilde{b}_{1}(y) D^{\alpha_{2}} \tilde{b}_{2}(y)}{|x-y|^{m}} K_{t}(x, y) f_{1}(y) d y \\
& +\int_{R^{n}} \frac{\prod_{j=1}^{2} R_{m_{j}+1}\left(\tilde{b}_{j} ; x, y\right)}{|x-y|^{m}} K_{t}(x, y) f_{2}(y) d y \\
= & A_{t_{Q}} T\left(\frac{\prod_{j=1}^{2} R_{m_{j}}\left(\tilde{b}_{j} ; x, \cdot\right)}{|x-\cdot|^{m}} f_{1}\right) \\
& -A_{t_{Q}} T\left(\sum_{\left|\alpha_{1}\right|=m_{1}} \frac{1}{\alpha_{1} !} \frac{R_{m_{2}}\left(\tilde{b}_{2} ; x, \cdot\right)(x-\cdot)^{\alpha_{1}} D^{\alpha_{1}} \tilde{b}_{1}}{|x-\cdot|^{m}} f_{1}\right) \\
& -A_{t_{Q}} T\left(\sum_{\left|\alpha_{2}\right|=m_{2}} \frac{1}{\alpha_{2} !} \frac{R_{m_{1}}\left(\tilde{b}_{1} ; x, \cdot\right)(x-\cdot)^{\alpha_{2}} D^{\alpha_{2}} \tilde{b}_{2}}{\mid x-\cdot f^{m}} f_{1}\right) \\
& +A_{t_{Q}} T\left(\sum_{\left|\alpha_{1}\right|=m_{1},\left|\alpha_{2}\right|=m_{2}} \frac{1}{\alpha_{1} ! \alpha_{2} !} \frac{(x-\cdot)^{\alpha_{1}+\alpha_{2}} D^{\alpha_{1}} \tilde{b}_{1} D^{\alpha_{2}} \tilde{b}_{2}}{\mid x-\cdot f^{m}} f_{1}\right) \\
& +A_{t_{Q}} T\left(\frac{\prod_{j=1}^{2} R_{m_{j}+1}\left(\tilde{b}_{j} ; x, \cdot\right)}{|x-\cdot|^{m}} f_{2}\right),
\end{aligned}
$$

then

$$
\begin{aligned}
& {\left[\left.\frac{1}{|Q|} \int_{Q}|| T^{b}(f)(x)\right|^{r}-\left|A_{t_{Q}} T^{b}(f)(x)\right|^{r} \mid d x\right]^{1 / r}} \\
& \leq\left[\frac{1}{|Q|} \int_{Q}\left|T^{b}(f)(x)-A_{t_{Q}} T^{b}(f)(x)\right|^{r} d x\right]^{1 / r} \\
& \leq\left[\frac{C}{|Q|} \int_{Q}\left|T\left(\frac{\prod_{j=1}^{2} R_{m_{j}}\left(\tilde{b}_{j} ; x, \cdot\right)}{|x-\cdot|^{m}} f_{1}\right)\right|^{r} d x\right]^{1 / r} \\
& +\left[\frac{C}{|Q|} \int_{Q}\left|T\left(\sum_{\left|\alpha_{1}\right|=m_{1}} \frac{R_{m_{2}}\left(\tilde{b}_{2} ; x, \cdot\right)(x-\cdot)^{\alpha_{1}} D^{\alpha_{1}} \tilde{b}_{1}}{|x-\cdot|^{m}} f_{1}\right)\right|^{r} d x\right]^{1 / r} \\
& +\left[\frac{C}{|Q|} \int_{Q}\left|T\left(\sum_{\left|\alpha_{2}\right|=m_{2}} \frac{R_{m_{1}}\left(\tilde{b}_{1} ; x, \cdot\right)(x-\cdot)^{\alpha_{2}} D^{\alpha_{2}} \tilde{b}_{2}}{|x-\cdot|^{m}} f_{1}\right)\right|^{r} d x\right]^{1 / r} \\
& +\left[\frac{C}{|Q|} \int_{Q}\left|T\left(\sum_{\left|\alpha_{1}\right|=m_{1},\left|\alpha_{2}\right|=m_{2}} \int_{Q} \frac{(x-\cdot)^{\alpha_{1}+\alpha_{2}} D^{\alpha_{1}} \tilde{b}_{1} D^{\alpha_{2}} \tilde{b}_{2}}{|x-\cdot|^{m}} f_{1}\right)\right|^{r} d x\right]^{1 / r} \\
& +\left[\frac{C}{|Q|} \int_{Q}\left|A_{t_{Q}} T\left(\frac{\prod_{j=1}^{2} R_{m_{j}}\left(\tilde{b}_{j} ; x, \cdot\right)}{|x-\cdot|^{m}} f_{1}\right)\right|^{r} d x\right]^{1 / r}
\end{aligned}
$$




$$
\begin{aligned}
& +\left[\frac{C}{|Q|} \int_{Q}\left|A_{t_{Q}} T\left(\sum_{\left|\alpha_{1}\right|=m_{1}} \frac{1}{\alpha_{1} !} \frac{R_{m_{2}}\left(\tilde{b}_{2} ; x, \cdot\right)(x-\cdot)^{\alpha_{1}} D^{\alpha_{1}} \tilde{b}_{1}}{|x-\cdot|^{m}} f_{1}\right)\right|^{r} d x\right]^{1 / r} \\
& +\left[\frac{C}{|Q|} \int_{Q}\left|A_{t_{Q}} T\left(\sum_{\left|\alpha_{2}\right|=m_{2}} \frac{1}{\alpha_{2} !} \frac{R_{m_{1}}\left(\tilde{b}_{1} ; x, \cdot\right)(x-\cdot)^{\alpha_{2}} D^{\alpha_{2}} \tilde{b}_{2}}{|x-\cdot|^{m}} f_{1}\right)\right|^{r} d x\right]^{1 / r} \\
& +\left[\frac{C}{|Q|} \int_{Q}\left|A_{t_{Q}} T\left(\sum_{\left|\alpha_{1}\right|=m_{1},\left|\alpha_{2}\right|=m_{2}} \frac{1}{\alpha_{1} ! \alpha_{2} !} \frac{(x-\cdot)^{\alpha_{1}+\alpha_{2}} D^{\alpha_{1}} \tilde{b}_{1} D^{\alpha_{2}} \tilde{b}_{2}}{|x-\cdot|^{m}} f_{1}\right)\right|^{r} d x\right]^{1 / r} \\
& +\left[\frac{C}{|Q|} \int_{Q}\left|\left(T-A_{t_{Q}} T\right)\left(\frac{\prod_{j=1}^{2} R_{m_{j}+1}\left(\tilde{b}_{j} ; x, \cdot\right)}{|x-\cdot|^{m}} f_{2}\right)\right|^{r} d x\right]^{1 / r} \\
& :=I_{1}+I_{2}+I_{3}+I_{4}+I_{5}+I_{6}+I_{7}+I_{8}+I_{9} .
\end{aligned}
$$

Now, let us estimate $I_{1}, I_{2}, I_{3}, I_{4}, I_{5}, I_{6}, I_{7}, I_{8}$ and $I_{9}$, respectively. First, for $x \in Q$ and $y \in \tilde{Q}$, by Lemma 1 , we get

$$
R_{m}\left(\tilde{b}_{j} ; x, y\right) \leq C|x-y|^{m} \sum_{\left|\alpha_{j}\right|=m}\left\|D^{\alpha_{j}} b_{j}\right\|_{B M O}
$$

by Lemma 2 and the weak type $(1,1)$ of $T$ (Lemma 4 ), we obtain

$$
\begin{aligned}
I_{1} & \leq C \prod_{j=1}^{2}\left(\sum_{\left|\alpha_{j}\right|=m_{j}}\left\|D^{\alpha_{j}} b_{j}\right\|_{B M O}\right)\left(\frac{1}{|Q|} \int_{R^{n}}\left|T\left(f_{1}\right)(x)\right|^{r} d x\right)^{1 / r} \\
& \leq C \prod_{j=1}^{2}\left(\sum_{\left|\alpha_{j}\right|=m_{j}}\left\|D^{\alpha_{j}} b_{j}\right\|_{B M O}\right)|Q|^{-1} \frac{\left\|T\left(f_{1}\right) \chi_{Q}\right\|_{L^{r}}}{|Q|^{1 / r-1}} \\
& \leq C \prod_{j=1}^{2}\left(\sum_{\left|\alpha_{j}\right|=m_{j}}\left\|D^{\alpha_{j}} b_{j}\right\|_{B M O}\right)|Q|^{-1}\left\|T\left(f_{1}\right)\right\|_{W L^{1}} \\
& \leq C \prod_{j=1}^{2}\left(\sum_{\left|\alpha_{j}\right|=m_{j}}\left\|D^{\alpha_{j}} b_{j}\right\|_{B M O}\right)|\tilde{Q}|^{-1}\left\|f_{1}\right\|_{L^{1}} \\
& \leq C \prod_{j=1}^{2}\left(\sum_{\left|\alpha_{j}\right|=m_{j}}\left\|D^{\alpha_{j}} b_{j}\right\|_{B M O}\right) M(f)(\tilde{x}) .
\end{aligned}
$$

For $I_{2}$, we get, by Lemma 2 and the generalized Hölder inequality,

$$
\begin{aligned}
I_{2} & \leq C \sum_{\left|\alpha_{2}\right|=m_{2}}\left\|D^{\alpha_{2}} b_{2}\right\|_{B M O} \sum_{\left|\alpha_{1}\right|=m_{1}}\left(\frac{1}{|Q|} \int_{R^{n}}\left|T\left(D^{\alpha_{1}} \tilde{b}_{1} f_{1}\right)(x)\right|^{r} d x\right)^{1 / r} \\
& \leq C \sum_{\left|\alpha_{2}\right|=m_{2}}\left\|D^{\alpha_{2}} b_{2}\right\|_{B M O} \sum_{\left|\alpha_{1}\right|=m_{1}}|Q|^{-1} \frac{\left\|T\left(D^{\alpha_{1}} \tilde{b}_{1} f_{1}\right) \chi_{Q}\right\|_{L^{r}}}{|Q|^{1 / r-1}} \\
& \leq C \sum_{\left|\alpha_{2}\right|=m_{2}}\left\|D^{\alpha_{2}} b_{2}\right\|_{B M O} \sum_{\left|\alpha_{1}\right|=m_{1}}|Q|^{-1}\left\|T\left(D^{\alpha_{1}} \tilde{b}_{1} f_{1}\right)\right\|_{W L^{1}} \\
& \leq C \sum_{\left|\alpha_{2}\right|=m_{2}}\left\|D^{\alpha_{2}} b_{2}\right\|_{B M O} \sum_{\left|\alpha_{1}\right|=m_{1}}|\tilde{Q}|^{-1}\left\|D^{\alpha_{1}} \tilde{b}_{1} f_{1}\right\|_{L^{1}}
\end{aligned}
$$




$$
\begin{aligned}
& \leq C \sum_{\left|\alpha_{2}\right|=m_{2}}\left\|D^{\alpha_{2}} b_{2}\right\|_{B M O} \sum_{\left|\alpha_{1}\right|=m_{1}}\left\|D^{\alpha_{1}} b_{1}-\left(D^{\alpha} b_{1}\right)_{\tilde{Q}}\right\|_{\exp L, \tilde{Q}}\|f\|_{L(\log L), \tilde{Q}} \\
& \leq C \prod_{j=1}^{2}\left(\sum_{\left|\alpha_{j}\right|=m_{j}}\left\|D^{\alpha_{j}} b_{j}\right\|_{B M O}\right) M^{2}(f)(\tilde{x}) .
\end{aligned}
$$

For $I_{3}$, similar to the proof of $I_{2}$, we get

$$
I_{3} \leq C \prod_{j=1}^{2}\left(\sum_{|\alpha|=m_{j}}\left\|D^{\alpha} b_{j}\right\|_{B M O}\right) M^{2}(f)(\tilde{x}) .
$$

Similarly, for $I_{4}$, taking $r, r_{1}, r_{2} \geq 1$ such that $1 / r=1 / r_{1}+1 / r_{2}$, we obtain, by Lemma 3 and the generalized Hölder inequality,

$$
\begin{aligned}
I_{4} & \leq C \sum_{\left|\alpha_{1}\right|=m_{1},\left|\alpha_{2}\right|=m_{2}}\left(\frac{1}{|Q|} \int_{R^{n}}\left|T\left(D^{\alpha_{1}} \tilde{b}_{1} D^{\alpha_{2}} \tilde{b}_{2} f_{1}\right)(x)\right|^{r} d x\right)^{1 / r} \\
& \leq C \sum_{\left|\alpha_{1}\right|=m_{1},\left|\alpha_{2}\right|=m_{2}}|Q|^{-1} \frac{\left\|T\left(D^{\alpha_{1}} \tilde{b}_{1} D^{\alpha_{2}} \tilde{b}_{2} f_{1}\right) \chi_{Q}\right\|_{L^{r}}}{|Q|^{1 / r-1}} \\
& \leq C \sum_{\left|\alpha_{1}\right|=m_{1},\left|\alpha_{2}\right|=m_{2}}|Q|^{-1}\left\|T\left(D^{\alpha_{1}} \tilde{b}_{1} D^{\alpha_{2}} \tilde{b}_{2} f_{1}\right)\right\|_{W L^{1}} \\
& \leq C \sum_{\left|\alpha_{1}\right|=m_{1},\left|\alpha_{2}\right|=m_{2}}|Q|^{-1}\left\|D^{\alpha_{1}} \tilde{b}_{1} D^{\alpha_{2}} \tilde{b}_{2} f_{1}\right\|_{L^{1}} \\
& \leq C \sum_{\left|\alpha_{1}\right|=m_{1},\left|\alpha_{2}\right|=m_{2}} \prod_{j=1}^{2}\left\|D^{\alpha_{j}} b_{j}-\left(D^{\alpha_{j}} b_{j}\right)_{\tilde{Q}}\right\|_{\exp L^{r_{j}, \tilde{Q}} \cdot\|f\|_{L(\log L)^{1 / r}, \tilde{Q}}} \\
& \leq C \prod_{j=1}^{2}\left(\sum_{|\alpha|=m_{j}}\left\|D^{\alpha} b_{j}\right\|_{B M O}\right) M^{3}(f)(\tilde{x}) .
\end{aligned}
$$

For $I_{5}, I_{6}, I_{7}$ and $I_{8}$, by Lemma 6 , we get

$$
\begin{aligned}
I_{5}+I_{6}+I_{7}+I_{8} \leq & C \prod_{j=1}^{2}\left(\sum_{\left|\alpha_{j}\right|=m_{j}}\left\|D^{\alpha_{j}} b_{j}\right\|_{B M O}\right) \frac{1}{|Q|} \int_{Q}\left|A_{t_{Q}} T\left(f_{1}\right)(x)\right| d x \\
& +C \sum_{\left|\alpha_{2}\right|=m_{2}}\left\|D^{\alpha_{2}} b_{2}\right\|_{B M O} \sum_{\left|\alpha_{1}\right|=m_{1}} \frac{1}{|Q|} \int_{Q}\left|A_{t_{Q}} T\left(D^{\alpha_{1}} \tilde{b}_{1} f_{1}\right)(x)\right| d x \\
& +C \sum_{\left|\alpha_{1}\right|=m_{1}}\left\|D^{\alpha_{1}} b_{1}\right\|_{B M O} \sum_{\left|\alpha_{2}\right|=m_{2}} \frac{1}{|Q|} \int_{Q}\left|A_{t_{Q}} T\left(D^{\alpha_{2}} \tilde{b}_{2} f_{1}\right)(x)\right| d x \\
& +C \sum_{\left|\alpha_{1}\right|=m_{1},\left|\alpha_{2}\right|=m_{2}} \frac{1}{|Q|} \int_{Q}\left|A_{t_{Q}} T\left(D^{\alpha_{1}} \tilde{b}_{1} D^{\alpha_{2}} \tilde{b}_{2} f_{1}\right)(x)\right| d x \\
\leq & C \prod_{j=1}^{2}\left(\sum_{|\alpha|=m_{j}}\left\|D^{\alpha} b_{j}\right\|_{B M O}\right) M^{3}(f)(\tilde{x}) .
\end{aligned}
$$


For $I_{9}$, we write

$$
\begin{aligned}
(T & \left.-A_{t_{Q}} T\right)\left(\frac{\prod_{j=1}^{2} R_{m_{j}+1}\left(\tilde{b}_{j} ; x, \cdot\right)}{|x-\cdot|^{m}} f_{2}\right) \\
= & \int_{R^{n}} \frac{\prod_{j=1}^{2} R_{m_{j}+1}\left(\tilde{b}_{j} ; x, y\right)}{|x-y|^{m}}\left(K(x, y)-K_{t}(x, y)\right) f_{2}(y) d y \\
= & \int_{R^{n}} \frac{\prod_{j=1}^{2} R_{m_{j}}\left(\tilde{b}_{j} ; x, y\right)}{|x-y|^{m}}\left(K(x, y)-K_{t}(x, y)\right) f_{2}(y) d y \\
& -\sum_{\left|\alpha_{1}\right|=m_{1}} \frac{1}{\alpha_{1} !} \int_{R^{n}} \frac{D^{\alpha_{1}} \tilde{b}_{1}(y)(x-y)^{\alpha_{1}} R_{m_{2}}\left(\tilde{b}_{2} ; x, y\right)}{|x-y|^{m}}\left(K(x, y)-K_{t}(x, y)\right) f_{2}(y) d y \\
& -\sum_{\left|\alpha_{2}\right|=m_{2}} \frac{1}{\alpha_{2} !} \int_{R^{n}} \frac{D^{\alpha_{2}} \tilde{b}_{2}(y)(x-y)^{\alpha_{2}} R_{m_{1}}\left(\tilde{b}_{1} ; x, y\right)}{|x-y|^{m}}\left(K(x, y)-K_{t}(x, y)\right) f_{2}(y) d y \\
& +\sum_{\left|\alpha_{1}\right|=m_{1},\left|\alpha_{2}\right|=m_{2}} \frac{1}{\alpha_{1} ! \alpha_{2} !} \int_{R^{n}} \frac{D^{\alpha_{1}} \tilde{b}_{1}(y) D^{\alpha_{2}} \tilde{b}_{2}(y)(x-y)^{\alpha_{1}+\alpha_{2}}}{|x-y|^{m}}\left(K(x, y)-K_{t}(x, y)\right) f_{2}(y) d y \\
= & I_{9}^{(1)}+I_{9}^{(2)}+I_{9}^{(3)}+I_{9}^{(4)} .
\end{aligned}
$$

By Lemma 1 and the following inequality (see [15])

$$
\left|b_{Q_{1}}-b_{Q_{2}}\right| \leq C \log \left(\left|Q_{2}\right| /\left|Q_{1}\right|\right)\|b\|_{B M O} \quad \text { for } Q_{1} \subset Q_{2},
$$

we know that for $x \in Q$ and $y \in 2^{k+1} \tilde{Q} \backslash 2^{k} \tilde{Q}$,

$$
\begin{aligned}
\left|R_{m}(\tilde{b} ; x, y)\right| & \leq C|x-y|^{m} \sum_{|\alpha|=m}\left(\left\|D^{\alpha} b\right\|_{B M O}+\left|\left(D^{\alpha} b\right)_{\tilde{Q}(x, y)}-\left(D^{\alpha} b\right)_{\tilde{Q}}\right|\right) \\
& \leq C k|x-y|^{m} \sum_{|\alpha|=m}\left\|D^{\alpha} b\right\|_{B M O} .
\end{aligned}
$$

Note that $|x-y| \geq d=t^{1 / 2}$ and $|x-y| \sim\left|x_{0}-y\right|$ for $x \in Q$ and $y \in R^{n} \backslash \tilde{Q}$. By the conditions on $K$ and $K_{t}$, we obtain

$$
\begin{aligned}
\left|I_{9}^{(1)}\right| & =\sum_{k=0}^{\infty} \int_{2^{k+1} \tilde{Q} \backslash 2^{k} \tilde{Q}} \frac{\prod_{j=1}^{2}\left|R_{m_{j}}\left(\tilde{b}_{j} ; x, y\right)\right|}{|x-y|^{m}}\left|K(x, y)-K_{t}(x, y)\right||f(y)| d y \\
& \leq C \prod_{j=1}^{2}\left(\sum_{|\alpha|=m_{j}}\left\|D^{\alpha} b_{j}\right\|_{B M O}\right) \sum_{k=0}^{\infty} \int_{2^{k+1} \tilde{Q} \backslash 2^{k} \tilde{Q}} k^{2} \frac{d^{\delta}}{\left|x_{0}-y\right|^{n+\delta}}|f(y)| d y \\
& \leq C \prod_{j=1}^{2}\left(\sum_{|\alpha|=m_{j}}\left\|D^{\alpha} b_{j}\right\|_{B M O}\right) \sum_{k=1}^{\infty} k^{2} 2^{-\delta k} \frac{1}{\left|2^{k} \tilde{Q}\right|} \int_{2^{k} \tilde{Q}}|f(y)| d y \\
& \leq C \prod_{j=1}^{2}\left(\sum_{|\alpha|=m_{j}}\left\|D^{\alpha} b_{j}\right\|_{B M O}\right) M(f)(\tilde{x}) .
\end{aligned}
$$


For $I_{9}^{(2)}$, we get, by the generalized Hölder inequality,

$$
\begin{aligned}
\left|I_{9}^{(2)}\right| \leq & C\left(\sum_{\left|\alpha_{2}\right|=m_{2}}\left\|D^{\alpha_{2}} b_{2}\right\|_{B M O}\right) \sum_{\left|\alpha_{1}\right|=m_{1}} \sum_{k=0}^{\infty} \int_{2^{k+1} \tilde{Q} \backslash 2^{k} \tilde{Q}} \frac{k d^{\delta}}{\left|x_{0}-y\right|^{n+\delta}}\left|D^{\alpha_{1}} \tilde{b}_{1}(y)\right| f f(y) \mid d y \\
\leq & C\left(\sum_{\left|\alpha_{2}\right|=m_{2}}\left\|D^{\alpha_{2}} b_{2}\right\|_{B M O}\right) \\
& \times \sum_{\left|\alpha_{1}\right|=m_{1}} \sum_{k=1}^{\infty} k 2^{-\delta k}\left\|D^{\alpha_{1}} b_{1}-\left(D^{\alpha_{1}} b_{1}\right)_{\tilde{Q}}\right\|_{\exp L, 2^{k} \tilde{Q}}\|f\|_{L(\log L), 2^{k} \tilde{Q}} \\
\leq & C \prod_{j=1}^{2}\left(\sum_{|\alpha|=m_{j}}\left\|D^{\alpha} b_{j}\right\|_{B M O}\right) M^{2}(f)(\tilde{x}) .
\end{aligned}
$$

Similarly,

$$
\left|I_{9}^{(3)}\right| \leq C \prod_{j=1}^{2}\left(\sum_{|\alpha|=m_{j}}\left\|D^{\alpha} b_{j}\right\|_{B M O}\right) M^{2}(f)(\tilde{x}) .
$$

For $I_{9}^{(4)}$, taking $r, r_{1}, r_{2} \geq 1$ such that $1 / r=1 / r_{1}+1 / r_{2}$, by Lemma 3 and the generalized Hölder inequality, we get

$$
\begin{aligned}
\left|I_{9}^{(4)}\right| & \leq C \sum_{\left|\alpha_{1}\right|=m_{1},\left|\alpha_{2}\right|=m_{2}} \sum_{k=0}^{\infty} \int_{2^{k+1} \tilde{Q} \backslash 2^{k} \tilde{Q}} \frac{d^{\delta}}{\left|x_{0}-y\right|^{n+\delta}}\left|D^{\alpha_{1}} \tilde{b}_{1}(y)\right|\left|D^{\alpha_{2}} \tilde{b}_{2}(y)\right||f(y)| d y \\
& \leq C \sum_{\left|\alpha_{1}\right|=m_{1},\left|\alpha_{2}\right|=m_{2}} \sum_{k=1}^{\infty} \prod_{j=1}^{2}\left\|D^{\alpha_{j}} b_{j}-\left(D^{\alpha_{j}} b_{j}\right)_{\tilde{Q}}\right\|_{\exp L^{r_{j}, 2^{k} \tilde{Q}}\|f\|_{L(\log L)^{1 / r}, 2^{k} \tilde{Q}}} \\
& \leq C \prod_{j=1}^{2}\left(\sum_{|\alpha|=m_{j}}\left\|D^{\alpha} b_{j}\right\|_{B M O}\right) M^{3}(f)(\tilde{x}) .
\end{aligned}
$$

Thus

$$
\left|I_{5}\right| \leq C \prod_{j=1}^{2}\left(\sum_{|\alpha|=m_{j}}\left\|D^{\alpha} b_{j}\right\|_{B M O}\right) M^{3}(f)(\tilde{x}) .
$$

This completes the proof of Theorem 1 .

By Theorem 1 and the $L^{p}(w)$-boundedness of $M^{l+1}$, we may obtain the conclusions of Theorem 2. By Theorem 1 and $[16,17]$, we may obtain the conclusions of Theorem 3.

\section{Applications}

In this section we shall apply Theorems 1, 2 and 3 of the paper to the holomorphic functional calculus of linear elliptic operators. First, we review some definitions regarding the holomorphic functional calculus (see [9]). Given $0 \leq \theta<\pi$, define

$$
S_{\theta}=\{z \in C:|\arg (z)| \leq \theta\} \cup\{0\}
$$


and its interior by $S_{\theta}^{0}$. Set $\tilde{S}_{\theta}=S_{\theta} \backslash\{0\}$. A closed operator $L$ on some Banach space $E$ is said to be of type $\theta$ if its spectrum $\sigma(L) \subset S_{\theta}$ and if for every $v \in(\theta, \pi]$, there exists a constant $C_{v}$ such that

$$
|\eta|\left\|(\eta I-L)^{-1}\right\| \leq C_{v}, \quad \eta \notin \tilde{S}_{\theta} .
$$

For $v \in(0, \pi]$, let

$$
H_{\infty}\left(S_{\mu}^{0}\right)=\left\{f: S_{\theta}^{0} \rightarrow C: f \text { is holomorphic and }\|f\|_{L^{\infty}}<\infty\right\} \text {, }
$$

where $\|f\|_{L^{\infty}}=\sup \left\{|f(z)|: z \in S_{\mu}^{0}\right\}$. Set

$$
\Psi\left(S_{\mu}^{0}\right)=\left\{g \in H_{\infty}\left(S_{\mu}^{0}\right): \exists s>0, \exists c>0 \text { such that }|g(z)| \leq c \frac{|z|^{s}}{1+|z|^{2 s}}\right\} .
$$

If $L$ is of type $\theta$ and $g \in H_{\infty}\left(S_{\mu}^{0}\right)$, we define $g(L) \in L(E)$ by

$$
g(L)=-(2 \pi i)^{-1} \int_{\Gamma}(\eta I-L)^{-1} g(\eta) d \eta
$$

where $\Gamma$ is the contour $\left\{\xi=r e^{ \pm i \phi}: r \geq 0\right\}$ parameterized clockwise around $S_{\theta}$ with $\theta<\phi<\mu$. If, in addition, $L$ is one-to-one and has a dense range, then, for $f \in H_{\infty}\left(S_{\mu}^{0}\right)$,

$$
f(L)=[h(L)]^{-1}(f h)(L),
$$

where $h(z)=z(1+z)^{-2}$. L is said to have a bounded holomorphic functional calculus on the sector $S_{\mu}$ if

$$
\|g(L)\| \leq N\|g\|_{L^{\infty}}
$$

for some $N>0$ and for all $g \in H_{\infty}\left(S_{\mu}^{0}\right)$.

Now, let $L$ be a linear operator on $L^{2}\left(R^{n}\right)$ with $\theta<\pi / 2$ so that $(-L)$ generates a holomorphic semigroup $e^{-z L}, 0 \leq|\arg (z)|<\pi / 2-\theta$. Applying Theorem 6 of [9], we get the following.

Theorem 4 Assume the following conditions are satisfied:

(i) The holomorphic semigroup $e^{-z L}, 0 \leq|\arg (z)|<\pi / 2-\theta$ is represented by the kernels $a_{z}(x, y)$ which satisfy, for all $v>\theta$, an upper bound

$$
\left|a_{z}(x, y)\right| \leq c_{\nu} h_{|z|}(x, y)
$$

for $x, y \in R^{n}$, and $0 \leq|\arg (z)|<\pi / 2-\theta$, where $h_{t}(x, y)=C t^{-n / 2} s\left(|x-y|^{2} / t\right)$ and s is a positive, bounded and decreasing function satisfying

$$
\lim _{r \rightarrow \infty} r^{n+\epsilon} s\left(r^{2}\right)=0 .
$$


(ii) The operator $L$ has a bounded holomorphic functional calculus in $L^{2}\left(R^{n}\right)$, that is, for all $v>\theta$ and $g \in H_{\infty}\left(S_{\mu}^{0}\right)$, the operator $g(L)$ satisfies

$$
\|g(L)(f)\|_{L^{2}} \leq c_{v}\|g\|_{L^{\infty}}\|f\|_{L^{2}}
$$

Then, for $D^{\alpha} b_{j} \in B M O\left(R^{n}\right)$ for all $\alpha$ with $|\alpha|=m_{j}$ and $j=1, \ldots, l$, the multilinear operator $g(L)^{b}$ associated to $g(L)$ and $b_{j}$ satisfies:

(a) For $0<r<1$ and $\tilde{x} \in R^{n}$,

$$
M_{A}^{\#}\left(g(L)^{b}(f)\right)_{r}(\tilde{x}) \leq C \prod_{j=1}^{l}\left(\sum_{\left|\alpha_{j}\right|=m_{j}}\left\|D^{\alpha_{j}} b_{j}\right\|_{B M O}\right) M^{l+1}(f)(\tilde{x}) ;
$$

(b) $g(L)^{b}$ is bounded on $L^{p}(w)$ for any $1<p<\infty$ and $w \in A_{p}$, that is,

$$
\left\|g(L)^{b}(f)\right\|_{L^{p}(w)} \leq C \prod_{j=1}^{l}\left(\sum_{\left|\alpha_{j}\right|=m_{j}}\left\|D^{\alpha_{j}} b_{j}\right\|_{B M O}\right)\|f\|_{L^{p}(w)} ;
$$

(c) There exists a constant $C>0$ such that for all $\lambda>0$ and $w \in A_{1}$,

$$
w\left(\left\{x \in R^{n}:\left|g(L)^{b}(f)(x)\right|>\lambda\right\}\right) \leq C \int_{R^{n}} \frac{|f(x)|}{\lambda}\left[1+\log ^{+}\left(\frac{|f(x)|}{\lambda}\right)\right]^{l} w(x) d x .
$$

\section{Competing interests}

The authors declare that they have no competing interests.

\section{Authors' contributions}

The authors completed the paper together. They also read and approved the final manuscript.

\section{Acknowledgements}

Project supported by Hunan Provincial Natural Science Foundation of China (12JJ6003) and Scientific Research Fund of Hunan Provincial Education Departments (12K017).

\section{Received: 17 March 2013 Accepted: 12 August 2013 Published: 16 September 2013}

\section{References}

1. Cohen, J: A sharp estimate for a multilinear singular integral on $R^{n}$. Indiana Univ. Math. J. 30, 693-702 (1981)

2. Cohen, J, Gosselin, J: On multilinear singular integral operators on $R^{n}$. Stud. Math. 72, 199-223 (1982)

3. Cohen, J, Gosselin, J: A BMO estimate for multilinear singular integral operators. III. J. Math. 30, 445-465 (1986)

4. Coifman, R, Meyer, Y: Wavelets, Calderón-Zygmund and Multilinear Operators. Cambridge Studies in Advanced Math., vol. 48. Cambridge University Press, Cambridge (1997)

5. Coifman, RR, Rochberg, R, Weiss, G: Factorization theorems for Hardy spaces in several variables. Ann. Math. 103, $611-635(1976)$

6. Ding, Y, Lu, SZ: Weighted boundedness for a class rough multilinear operators. Acta Math. Sin. 17, 517-526 (2001)

7. Pérez, C, Trujillo-Gonzalez, R: Sharp weighted estimates for multilinear commutators. J. Lond. Math. Soc. 65, 672-692 (2002)

8. Hu, GE, Yang, DC: A variant sharp estimate for multilinear singular integral operators. Stud. Math. 141, 25-42 (2000)

9. Duong, XT, McIntosh, A: Singular integral operators with non-smooth kernels on irregular domains. Rev. Mat. Iberoam. 15, 233-265 (1999)

10. Liu, LZ: Sharp function boundedness for vector-valued multilinear singular integral operators with non-smooth kernels. J. Contemp. Math. Anal. 45, 185-196 (2010)

11. Liu, LZ: Multilinear singular integral operators on Triebel-Lizorkin and Lebesgue spaces. Bull. Malays. Math. Soc. 35 1075-1086 (2012)

12. Martell, JM: Sharp maximal functions associated with approximations of the identity in spaces of homogeneous type and applications. Stud. Math. 161, 113-145 (2004)

13. Deng, DG, Yan, LX: Commutators of singular integral operators with non-smooth kernels. Acta Math. Sci. 25, 137-144 (2005) 
14. Garcia-Cuerva, J, Rubio de Francia, JL: Weighted Norm Inequalities and Related Topics. North-Holland Mathematics Studies, vol. 116. North-Holland, Amsterdam (1985)

15. Stein, EM: Harmonic Analysis: Real Variable Methods, Orthogonality and Oscillatory Integrals. Princeton University Press, Princeton (1993)

16. Pérez, C: Endpoint estimate for commutators of singular integral operators. J. Funct. Anal. 128, 163-185 (1995)

17. Pérez, C, Pradolini, G: Sharp weighted endpoint estimates for commutators of singular integral operators. Mich. Math. J. 49, 23-37 (2001)

doi:10.1186/1029-242X-2013-439

Cite this article as: Gu and Cai: A sharp inequality for multilinear singular integral operators with non-smooth kernels. Journal of Inequalities and Applications 2013 2013:439.

Submit your manuscript to a SpringerOpen ${ }^{\circ}$ journal and benefit from:

- Convenient online submission

Rigorous peer review

- Immediate publication on acceptance

- Open access: articles freely available online

- High visibility within the field

- Retaining the copyright to your article

Submit your next manuscript at $>$ springeropen.com 